\title{
Detection of horizontal root fracture using four different protocols of cone-beam computed tomography
}

\section{Fernanda Cristina Sales SALINEIRO \\ Lucas Rodrigues PINHEIRO Oséas dos SANTOS JÚNIOR Marcelo Gusmão Paraiso CAVALCANTI}

Universidade de São Paulo - USP, School of Dentistry, Department of Stomatology, São Paulo, SP, Brazil.
Declaration of Interests: The authors certify that they have no commercial or associative interest that represents a conflict of interest in connection with the manuscript.

Corresponding Author:

Marcelo Gusmão Paraiso Cavalcanti

E-mail:mgpcaval@usp.br

DOI: 10.1590/1807-3107BOR-2015.vol29.0067

Submitted: Oct 29, 2014

Accepted for publication: Feb 06, 2015

Last revision: May 05, 2015

\begin{abstract}
The purpose of this study was to analyze four different cone-beam computed tomography (CBCT) protocols to identify horizontal root fractures (HRF) in endodontically treated teeth, with or without the presence of a metallic post. Thirty extracted single-rooted human premolars were treated endodontically. Afterwards, an observer induced a horizontal fracture in 15 teeth. Each tooth was inserted in an empty mandibular socket and submitted to a computed tomography scan taken with and without the metallic post. The acquisition followed four different protocols, with different fields of view (FOV) and voxel sizes, as follows: FOV 6 X16 cm/0.2 mm voxel; FOV 6X16 cm/0.25 mm voxel; FOV $8 X 8 \mathrm{~cm} / 0.2 \mathrm{~mm}$ voxel; FOV $8 X 8 \mathrm{~cm} / 0.25 \mathrm{~mm}$ voxel. Two observers checked all the acquisitions within a two-week interval, and the values of sensitivity, specificity, accuracy and kappa were calculated. The sensitivity, specificity and accuracy values were better for the $8 \times 8 \mathrm{~cm} / 0.2 \mathrm{~mm}$ and $16 \times 6 \mathrm{~cm} / 0.25 \mathrm{~mm}$ protocols, ranging from 0.60 to 0.86 and 0.53 to 0.80 , respectively. The intra- and interobserver concordance ranged from 0.65 to 0.72 . The protocols where FOV and voxel were proportional showed better results. The $8 \times 8 \mathrm{~cm} / 0.2 \mathrm{~mm}$ protocol had the least interference from the metallic artifact. All four protocols showed a decline in values in the presence of the metallic artifact.
\end{abstract}

Keywords: Cone-Beam Computed Tomography; Diagnostic Imaging; Tooth Fracture.

\section{Introduction}

Root fracture is a type of dental injury with the worst prognosis in dentistry. A correct diagnosis is essential for effective dental treatment, and this is accomplished by clinical and radiographic examination. ${ }^{1}$

The etiology of root fracture is multifactorial. In permanent teeth, the fracture generally occurs in both anterior teeth and posterior teeth, secondary to trauma or iatrogenic procedures. ${ }^{2}$

The diagnosis of root fracture is more difficult to perform correctly after the obturation and insertion of a metallic post. Two-dimensional techniques are not accurate to diagnose dental fractures, because the fractures may be masked, particularly if the projection angle is not perpendicular to the fracture line. ${ }^{3}$

The use of three-dimensional scans in dentistry has been increasing constantly. Although patients receive a higher dose of radiation than in 
periapical radiography, the quality of the images allows clearer visualization without superimposition of images, and thus enables a more specific diagnosis., ${ }^{4,5}$

Some authors mention difficulty in diagnosing horizontal root fractures (HRF) when the tooth has artifacts such as metallic restorations, gutta percha, a metallic post and a metallic core crown. ${ }^{4,5}$

There are several parameters that may influence the quality of cone-beam computed tomography (CBCT) examination, such as $x$-ray beam factors, size of field of view (FOV), detector type, and size of the reconstructed voxels. Variations in parameters associated to differences in CBCT units and imaging protocols in a specific unit may lead to differences in the resolution of images. ${ }^{4,6}$

Based on the influence of so many variations, the purpose of this study was to analyze four different CBCT protocols to identify HRFs in endodontically treated teeth, with or without a metallic post.

\section{Methodology}

\section{Preparation of Samples}

Thirty extracted single-rooted human premolar teeth with no root resorption, cracks, caries, or fractures were selected. The anatomic crowns of all the teeth were sectioned perpendicular to the long axis at the cementoenamel junction, using water-cooled diamond burs driven by an air turbine (300,000 rpm).

The Research Ethics Committee of our institution approved this research, designated by protocol number 186/2010, CAAE 0013.0.017.000-10.

The same observer, who was not involved in interpreting the images, performed the endodontic treatment for all the teeth, using Gates Glidden drills sizes \#2 and \#3, and \#40 and \#30, as well as Nitiflex files (Dentsply Maillefer, Ballaigues, Switzerland), obturation with a sealer (Pulp Canal Sealer; Sybron Endo, Orange, USA), and gutta-percha points (Dentsply Maillefer, Ballaigues, Switzerland). The observer removed the fillings from the root canals up to two-thirds of their length. Afterwards, a post was modeled within each root canal.

The observer induced a horizontal root fracture in 15 teeth by applying a mechanical force on their horizontal plane, with a hammer. The teeth were then placed on a soft foundation, as previously described by Costa et al., ${ }^{4,7}$ Hassan et al., ${ }^{8}$ and Wenzel and Kirkevang. ${ }^{9}$ Both fragments of each tooth root were then assembled and glued without displacement. According to the inclusion criteria, the four roots that broke into more than two fragments were replaced. The entire sample was kept hydrated during the process, except during the fracture induction procedure.

\section{Image Acquisition}

The mandible was immersed in a water-filled plastic recipient to simulate the attenuation of $x$-rays promoted by the soft tissues, as previously described by Costa et al.,7 Moreira et al., ${ }^{10}$ and Katsumata et al.11,12 A CBCT iCAT (Imaging Sciences International, Hatfield, USA) scan was performed for each tooth placed individually in the empty mandibular sockets of 30 human dry mandibles, to obtain four different acquisition protocols.

The limits of the imaging area (FOV) consisted of a 6-cm high and 16-cm diameter cylinder, and an 8 -cm high and 8-cm diameter cylinder.

The voxel sizes were: $0.2 \mathrm{~mm}$ and $0.25 \mathrm{~mm}$.

The acquisition protocols were (FOV/voxel):

- $6 \times 16 / 0.2 \mathrm{~mm}$

- $6 \times 16 / 0.25 \mathrm{~mm}$

- $8 \times 8 / 0.2 \mathrm{~mm}$

- $8 \times 8 / 0.25 \mathrm{~mm}$

The cobalt chromium (Co-Cr) metallic posts were then inserted into the root canals, and the teeth were scanned again following the same protocols. Accordingly, 30 CBCT scans of roots with a metallic post and an equal number of images without a metallic post (each group containing 15 teeth with a fracture and 15 without a fracture) were obtained for each protocol.

Two double-blinded observers, who were oral and maxillofacial radiologists, trained and calibrated for tomographic features of horizontal root fracture, analyzed 240 CBCT scans in each observation.

\section{Radiographic assessment}

After image acquisition, the data were imported into a specially designed open-source DICOM viewer for MacOS - OsiriX 3.8.1 version (Pixmeo, Geneva, Switzerland; http://www.osirix-viewer.com/). 
The observers interpreted the volume data using multiplanar reconstructed images (axial, coronal and sagittal), as well as both parasagittal and circumferential images. They had to identify the fracture and its location on the root surface (cervical, middle or apical). Incorrect locations were considered misdiagnoses. A software application (Randomness 1.5.2; Andrew Merenbach, Los Angeles, USA) randomized the sequence of observations.

The interpretation time was not restricted. All the observers used the same workstation to perform the analysis. The same images were analyzed repeatedly after a 2-week interval, so that the observers would not remember the previous image interpretation. This procedure was planned to evaluate intraobserver agreement.

\section{Data Analysis}

The presence of a fracture line was diagnosed by a dichotomous (yes/no) evaluation, as follows: 1) correct identification of a nonfractured root (true negative [TN]); 2) a fracture in a fractured root (true positive [TP]); 3) identification of a fracture in a nonfractured root (false positive [FP]); 4) failure to identify a fracture in a fractured root (false negative $[\mathrm{FN}])$. Then, the sensitivity $=[\mathrm{TP} /(\mathrm{TP}+\mathrm{FN})]$, the specificity $=[\mathrm{TN} /(\mathrm{TN}+\mathrm{FP})]$ and the accuracy $=[(\mathrm{TP}+\mathrm{TN}) /(\mathrm{TP}+\mathrm{FN}+\mathrm{TN})]$ were calculated. Statistical analyses were performed using the validity and kappa tests. The kappa coefficient was calculated to assess the degree of intra- and interobserver agreement, and scored as weak (0.20-0.39), moderate (0.40-0.59) or relevant (0.60-0.79). ${ }^{9}$ It allowed the agreement between the tomographic diagnosis and the gold standard to be checked. The level of significance was set at $=0.05$. The data were analyzed using SPSS software (v 17.0.0; SPSS Inc., Chicago, USA).

\section{Results}

The study included thirty premolar teeth, fifteen with root fracture and fifteen without root fracture. All the teeth were scanned twice, once with and once without a metallic post. Table 1 shows the results for sensitivity, specificity and accuracy in the samples observed, using all four protocols. Sensitivity values, in this case, proved to be the most appropriate examination to identify HRF.

The results for accuracy were represented as the total number of true positives and true negatives. Protocols 8x8/0.2 and 16x6/0.25 showed slightly better sensitivity and specificity values, compared with the others protocols, ranging from 0.60 to 0.86 for $8 \times 8 / 0.2$, and 0.80 to 0.53 for $16 \times 6 / 0.25$. The presence of metallic artifacts showed significant interference in all the protocols, in a homogeneous and similar manner. Thus, the sensitivity and specificity values were reduced in all four protocols.

Table 1. Sensitivity, specificity coefficients, accuracy rates and intraobserver agreement on HRF diagnosis in groups of teeth without and with metallic posts.

\begin{tabular}{|c|c|c|c|c|c|c|c|c|c|c|c|c|c|c|}
\hline \multirow{4}{*}{$\begin{array}{l}\text { Protocol } \\
\text { FOV/Voxel }\end{array}$} & \multicolumn{7}{|c|}{ Observer \# 1} & \multicolumn{7}{|c|}{ Observer \#2 } \\
\hline & \multicolumn{7}{|c|}{ Samples without metallic post } & \multicolumn{7}{|c|}{ Samples without metallic post } \\
\hline & \multicolumn{3}{|c|}{ First observation } & \multicolumn{3}{|c|}{ Second observation } & & \multicolumn{3}{|c|}{ First observation } & \multicolumn{3}{|c|}{ Second observation } & \\
\hline & $\mathrm{Se}$ & $S p$ & $A(\%)$ & $\mathrm{Se}$ & $\mathrm{Sp}$ & $A(\%)$ & Agr & $\mathrm{Se}$ & Sp & $A(\%)$ & $\mathrm{Se}$ & Sp & $A(\%)$ & Agr \\
\hline $8 \times 8 / 0.2$ & 0.40 & 0.46 & 43.33 & 0.40 & 0.60 & 50.00 & 0.65 & 0.60 & 0.46 & 53.33 & 0.73 & 0.60 & 66.67 & 0.38 \\
\hline $8 \times 8 / 0.25$ & 0.53 & 0.60 & 56.67 & 0.40 & 0.57 & 48.28 & 0.66 & 0.80 & 0.73 & 76.67 & 0.73 & 0.73 & 73.33 & 0.40 \\
\hline $6 \times 16 / 0.2$ & 0.73 & 0.61 & 67.86 & 0.60 & 0.46 & 53.85 & 0.65 & 0.86 & 0.60 & 73.33 & 0.60 & 0.66 & 63.33 & 0.45 \\
\hline $6 \times 16 / 0.25$ & 0.80 & 0.33 & 56.67 & 0.73 & 0.53 & 63.33 & 0.70 & 0.66 & 0.86 & 76.67 & 0.53 & 0.80 & 66.67 & 0.36 \\
\hline \multirow{3}{*}{$\begin{array}{l}\text { Protocol } \\
\text { FOV/Voxel }\end{array}$} & \multicolumn{7}{|c|}{ Samples with metallic post } & \multicolumn{7}{|c|}{ Samples with metallic post } \\
\hline & \multicolumn{3}{|c|}{ First observation } & \multicolumn{3}{|c|}{ Second observation } & & \multicolumn{3}{|c|}{ First observation } & \multicolumn{3}{|c|}{ Second observation } & \\
\hline & $\mathrm{Se}$ & $\mathrm{Sp}$ & $A(\%)$ & $\mathrm{Se}$ & $\mathrm{Sp}$ & $A(\%)$ & Agr & $\mathrm{Se}$ & $\mathrm{Sp}$ & $A(\%)$ & $\mathrm{Se}$ & Sp & $\mathrm{A}(\%)$ & Agr \\
\hline $8 \times 8 / 0.2$ & 0.26 & 0.53 & 40.00 & 0.26 & 0.46 & 36.67 & 0.63 & 0.86 & 0.60 & 73.33 & 0.73 & 0.73 & 73.33 & 0.59 \\
\hline $8 \times 8 / 0.25$ & 0.20 & 0.66 & 43.33 & 0.20 & 0.60 & 40.00 & 0.42 & 0.60 & 0.73 & 66.67 & 0.60 & 0.66 & 63.33 & 0.52 \\
\hline $6 \times 16 / 0.2$ & 0.33 & 0.60 & 46.67 & 0.26 & 0.60 & 43.33 & 0.22 & 0.80 & 0.86 & 83.33 & 0.66 & 0.73 & 70.00 & 0.33 \\
\hline $6 \times 16 / 0.25$ & 0.40 & 0.60 & 50.00 & 0.33 & 0.53 & 43.33 & 0.72 & 0.53 & 0.73 & 63.33 & 0.46 & 0.66 & 56.67 & 0.72 \\
\hline
\end{tabular}


The kappa values for intraobserver agreement in all the protocols ranged from 0.36 to 0.72 . Observer 1 and Observer 2 performed better in protocol $16 \times 6 / 0.25$, and showed the same result: 0.72 . All the kappa values may be considered good, according to Altman. ${ }^{13}$ The interobserver concordance presented better values in the $8 \times 8 / 0.2$ and $16 \times 6 / 0.25$ protocols. The results obtained ranged from 0.43 to 0.63 in the analysis of samples without a metallic post, versus 0.40 to 0.60 for samples with a metallic post (Table 2).

\section{Discussion}

According to Costa et al., ${ }^{4,7}$ there are different acquisition and post-acquisition protocols for three-dimensional examination in the same CBCT scanner. Different FOV sizes and acquisition times may influence the quality of a CT scan and the radiation dose delivered to the patient. The FOV selection is directly related to the voxel size and influences spatial and contrast resolution. A large FOV provides less resolution and contrast, compared with a small FOV., $, 7,8,14,15$

A smaller voxel size will not detect as many $x$-ray photons as a larger voxel would. A decrease in the number of photons acquired by a voxel would result in a decrease in the signal, leading, in turn, to an increase in noise, as suggested by Bechara et al. ${ }^{16}$ However, a larger voxel size results in lower spatial resolution, and should not be used if the scan serves to depict fine details. In our research, we used both a small-volume and a large-volume CBCT to perform the scans with two different voxels to monitor the interference of image noise. In our opinion, noise was manifested to a greater extent in images when the FOV/ voxel relationship was not proportional. This reduced the interval of confidence of the diagnosis, leading to intraobserver agreement at levels up to $0.22-0.40$.
Iikubo et al. ${ }^{17}$ concluded that the CBCT should be considered the most reliable imaging modality of choice for the diagnosis of HRF. Limited cone-beam CT (CBCT with a small FOV) has the highest sensitivity and diagnostic accuracy for detecting HRF among all radiographic modalities. In our study, we found better results when the parameters of the $\mathrm{CBCT}$ imaging acquisition protocol were proportional (FOV vs. voxel size). Accordingly, we obtained images with better resolution for diagnosis of horizontal root fractures (Figure 1A, 1C, 1E and 1G).

Kamburoğlu et al. ${ }^{18}$ found better results for CBCT with a smaller FOV, when detecting and locating HRF ex vivo. Different CBCT units using limited FOV and high resolution showed similar results in detecting simulated HRF ex vivo. Considering the lower radiation dose advantage, limited CBCT units can be used with the smallest available FOV for detecting suspected HRF. We agree that a smaller FOV may be better for detecting HRF, but our results showed that the small FOV showed better numbers when a smaller voxel size was preferred (Figure 1B, 1D, 1F and $1 \mathrm{H}$ ).

CBCT images can be corrupted by artifacts caused by root filling materials. These reduce the accuracy, sensitivity and specificity of the CBCT technique in detecting root fractures. ${ }^{4,78}$ In our study, we observed that the images could have been influenced by corruption in the presence of an artifact in all the image acquisition protocols. This was observed when we compared the sensitivity levels between the samples without metallic post $(0.40-0.80)$ and those with metallic post (0.20-0.40). Therefore, the protocol with the largest FOV and the smallest voxel size showed more dental metallic interference, corroborating the results by Hassan et al., ${ }^{8}$ Costa et al. ${ }^{4,7}$

Brito-Júnior et al. ${ }^{19}$ evaluated streaking artifacts produced by several root canal sealers on CBCT

Table 2. Intraobserver Concordance on Diagnosis of Horizontal Root Fracture in Teeth without and with Metallic Posts.

\begin{tabular}{lllll}
\hline Protocol & \multicolumn{2}{c}{ Observer \# 1 X Observer \# 1 } & \multicolumn{2}{c}{ Observer \#2 X Observer \#2 } \\
\cline { 2 - 5 } FOV/Noxel & Samples without metallic post & Samples with metallic post & Samples without metallic post & Samples with metallic post \\
\hline $8 \times 8 / 0.2$ & 0.65 & 0.63 & 0.38 & 0.59 \\
$8 \times 8 / 0.25$ & 0.66 & 0.42 & 0.40 & 0.52 \\
$16 \times 6 / 0.2$ & 0.65 & 0.22 & 0.45 & 0.33 \\
$16 \times 6 / 0.25$ & 0.70 & 0.72 & 0.36 & 0.72 \\
\hline
\end{tabular}



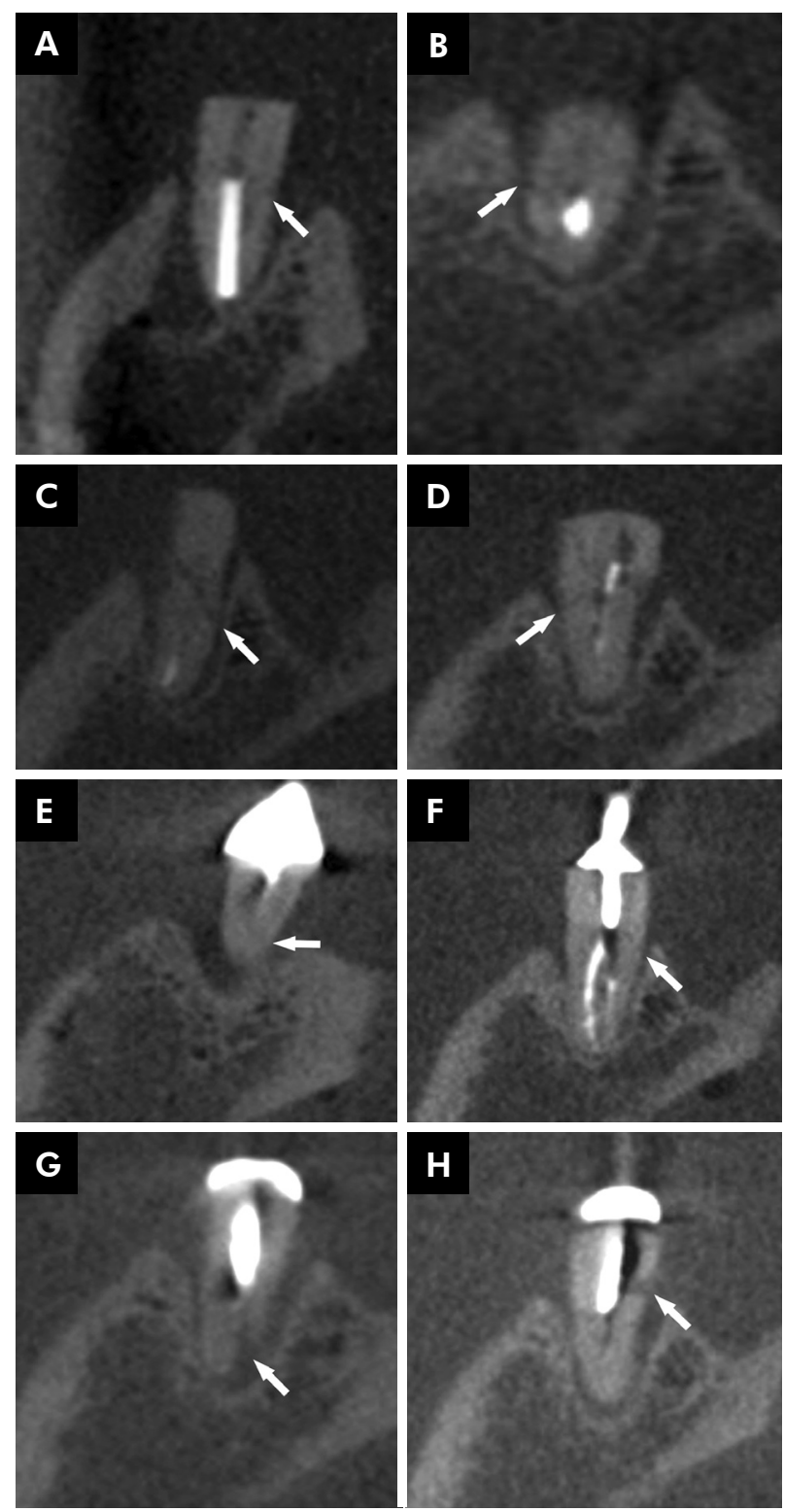

Samples with no root fracture and no metallic post; presence of a radiolucent image caused by an artifact (arrow) (A, 8x8/0.2 protocol and $B, 16 \times 6 / 0.2$ protocol).

Samples with a root fracture (arrow) and no metallic post (C, $8 \times 8 / 0.2$ protocol and $D, 16 \times 6 / 0.2$ protocol).

Samples with no horizontal root fracture and with a metallic post; presence of a radiolucent image caused by a metal artifact (arrow) ( $E, 8 \times 8 / 0.2$ protocol and $F, 16 \times 6 / 0.2$ protocol).

Samples with a root fracture (arrow) and a metallic post. (G, $8 \times 8 / 0.2$ protocol and $H, 16 \times 6 / 0.2$ protocol).

Figure 1. Sagittal CBCT images.

images with variations in voxel resolution. This study revealed that gutta-percha cones created distinct streaking artifacts on CBCT axial slices. The CBCT images also showed that some brands induced a higher number of artifacts than others, when a 0.20 mm voxel size was used. In some cases, we found imaging patterns without a metallic post, which suggested the presence of HRF. However, this was considered a false-positive, since the fracture did not occur in the sample. This artifact could have been produced by fillings (Figure 1E).

Acquisition protocols could result in the equipment delivering unacceptably high x-ray doses to patients, for certain imaging procedures. This is particularly important when children are exposed, considering that their exposure is more common in dental procedures, according to Horner et al. ${ }^{20} \mathrm{~A}$ larger FOV generates a number of images beyond the area of interest for diagnosis. As a result, adjacent tissue is unnecessarily exposed to radiation. The selection criteria and the parameters for each $\mathrm{CBCT}$ scan protocol should follow the respective clinical indication. All exposure to ionizing radiation should follow the ALARA (As Low As Reasonably Achievable) principle. ${ }^{8}$

According to our results, the sensitivity found in the observations of the samples with or without a metallic post in the $8 \times 8 / 0.2$ protocol was higher than that found in the $8 \times 8 / 0.25$ protocol. This could mean that the observer had a greater chance of identifying the HRF in the protocol with a smaller voxel and a smaller FOV. The same situation was observed in the $16 \times 6 / 0.25$ protocol, which showed higher sensitivity than that found in the 16x6/0.2 protocol, which had a larger voxel and a larger FOV. The lower sensitivity and specificity values were associated with the difficulty in detecting root fracture in the presence of a metal artifact. These values showed that FOV and voxel are directly proportional. Based on the results of this study, it is believed that a proportional protocol generates better images to diagnose horizontal root fracture. The overall results of a study by Costa et al. ${ }^{4,7}$ showed a more efficient diagnosis of HRF in protocols using a small-volume versus a large-volume CBCT scan.

\section{Conclusion}

The protocols where FOV and voxel size were more proportional $(6 \times 16 / 0.25 \mathrm{~mm}$ and $8 \times 8 / 0.20 \mathrm{~mm})$ showed better results. 
The $6 \times 16 / 0.2 \mathrm{~mm}$ was considered the worst protocol, both in the absence and in the presence of a metal artifact.

The 8X8/0.2 $\mathrm{mm}$ protocol showed less interference from a metal artifact. All four protocols showed a decline in values in the presence of a metal artifact.

\section{Acknowledgments}

Funding was provided by the $\mathrm{CNPq}$ (Conselho Nacional de Desenvolvimento Científico e Tecnológico),

\section{References}

1. Orhan K, Aksoy U, Kalender A. Cone-beam computed tomographic evaluation of spontaneously healed root fracture. J Endod. 2010 Sep;36(9):1584-7.

2. Testori T, Badino M, Castagnola M. Vertical root fractures in endodontically treated teeth: a clinical survey of 36 cases. J Endod. 1993 Feb:19(2):87-91.

3. Shintaku WH, Venturin JS, Noujeim M, Dove SB. Comparison between intraoral indirect and conventional film-based imaging for the detection of dental root fractures: an ex vivo study. Dent Traumatol. 2013 Dec;29(6):445-9.

4. Costa FF, Gaia BF, Umetsubo OS, Pinheiro LR, Tortamano IP, Cavalcanti MGP. Use of large-volume cone-beam computed tomography in identification and localization of horizontal root fracture in the presence and absence of intracanal metallic post. J Endod. 2012 Jun;38(6):856-9.

5. Perrella A, Lopes PML, Rocha RG, Fenyo-Pereira M, Cavalcanti MGP. Influence of dental metallic artifact from multislice CT in the assessment of simulated mandibular lesions. J Appl Oral Sci. 2010 Mar-Apr;18(2):149-54.

6. Librizzi ZT, Tadinada AS, Valiyaparambil JV, Lurie AG, Mallya SM. Cone-beam computed tomography to detect erosions of the temporomandibular joint: effect of field of view and voxel size on diagnostic efficacy and effective dose. Am J Orthod Dentofacial Orthop. 2011 Jul;140(1):e25-30.

7. Costa FF, Gaia BF, Umetsubo OS, Cavalcanti MGP. Detection of horizontal root fracture with small-volume cone-beam computed tomography in the presence and absence of intracanal metallic post. J Endod. 2011 Oct;37(10):1456-9.

8. Hassan B, Metska ME, Ozok AR, van der Stelt P, Wesselink PR. Detection of vertical root fractures in endodontically treated teeth by a cone beam computed tomography scan. J Endod. 2009 May;35(5):719-22.

9. Wenzel A, Kirkevang LL. High resolution charge-coupled device sensor vs medium resolution photostimulable phosphor plate digital receptors for detection of root fractures in vitro. Dent Traumatol. 2005 Feb;21(1):32-6.

10. Moreira CR, Sales MA, Lopes PM, Cavalcanti MG. Assessment of linear and angular measurements on three-dimensional cone-beam computed tomographic images. Oral Surg Oral Med Oral Pathol Oral Radiol Endod. 2009 Sep;108(3):430-6.
Brasília, Brazil, grant no. 303847/2009-3 (to Marcelo Gusmão Paraíso Cavalcanti); Universal Research Project grant no. 472895/2009-5 (to Marcelo Gusmão Paraíso Cavalcanti); Coordenação de Aperfeiçoamento de Pessoal de Nível Superior - CAPES (Master's scholarship to Oseas Santos Junior); and Fundação de Amparo à Pesquisa do Estado de São Paulo - FAPESP (PhD scholarship to Lucas Rodrigues Pinheiro).

11. Katsumata A, Hirukawa A, Okumura S, Naitoh M, Fujishita $\mathrm{M}$, Ariji E, et al. Effects of image artifacts on gray-value density in limited-volume cone-beam computerized tomography. Oral Surg Oral Med Oral Pathol Oral Radiol Endod. 2007 Dec;104(6):829-36.

12. Katsumata A, Hirukawa A, Okumura S, Naitoh M, Fujishita M, Ariji E, et al. Relationship between density variability and imaging volume size in cone-beam computerized tomographic scanning of the maxillofacial region: an in vitro study. Oral Surg Oral Med Oral Pathol Oral Radiol Endod. 2009 Mar;107(3):420-25.

13. Altman DG. Practical statistics for medical research. 1th ed. London: Chapman and Hall. 1991. 624 p.

14. American Association of Endodontists; American Academy of Oral and Maxillofacial Radiology. Use of cone-beam computed tomography in endodontics. Oral Surg Oral Med Oral Pathol Oral Radiol Endod. 2011 Feb;111(2):234-7.

15. Cavalcanti MGP. Cone beam computed tomographic imaging: perspective, challenges, and the impact of near-trend future applications. J Craniofac Surg. 2012 Jan;23(1):279-82.

16. Bechara B, McMahan CA, Moorei WS, Noujeim M, Geha H, Teixeira FB. Contrast-to-noise ratio difference in small field of view cone beam computed tomography machines. J Oral Sci. 2012 Sep;54(3):227-32.

17. Iikubo M, Kobayashi K, Mishima A, Shimoda S, Daimaruya $\mathrm{T}$, Igarashi $\mathrm{C}$, et al. Accuracy of intraoral radiography, multidetector helical CT, and limited cone-beam CT for the detection of horizontal tooth root fracture. Oral Surg Oral Med Oral Pathol Oral Radiol Endod. 2009 Nov;108(5):e70-4.

18. Kamburoğlu K, Önder B, Murat S, Avsever H, Yüksel S, Paksoy CS. Radiographic detection of artificially created horizontal root fracture using different cone beam CT units with small fields of view. Dentomaxillofac Radiol. 2013;42(4):20120261.

19. Brito-Júnior M, Santos LAN, Faria-e-Silva AL, Pereira RD, Sousa-Neto MD. Ex vivo evaluation of artifacts mimicking fracture lines on cone-beam computed tomography produced by different root canal sealers. Int Endod J. 2014 Jan;47(1):26-31.

20. Horner K, Jacobs R, Schulze R. Dental CBCT equipment and performance issues. Radiat Prot Dosimetry. 2013 Feb;153(2):212-8. 